
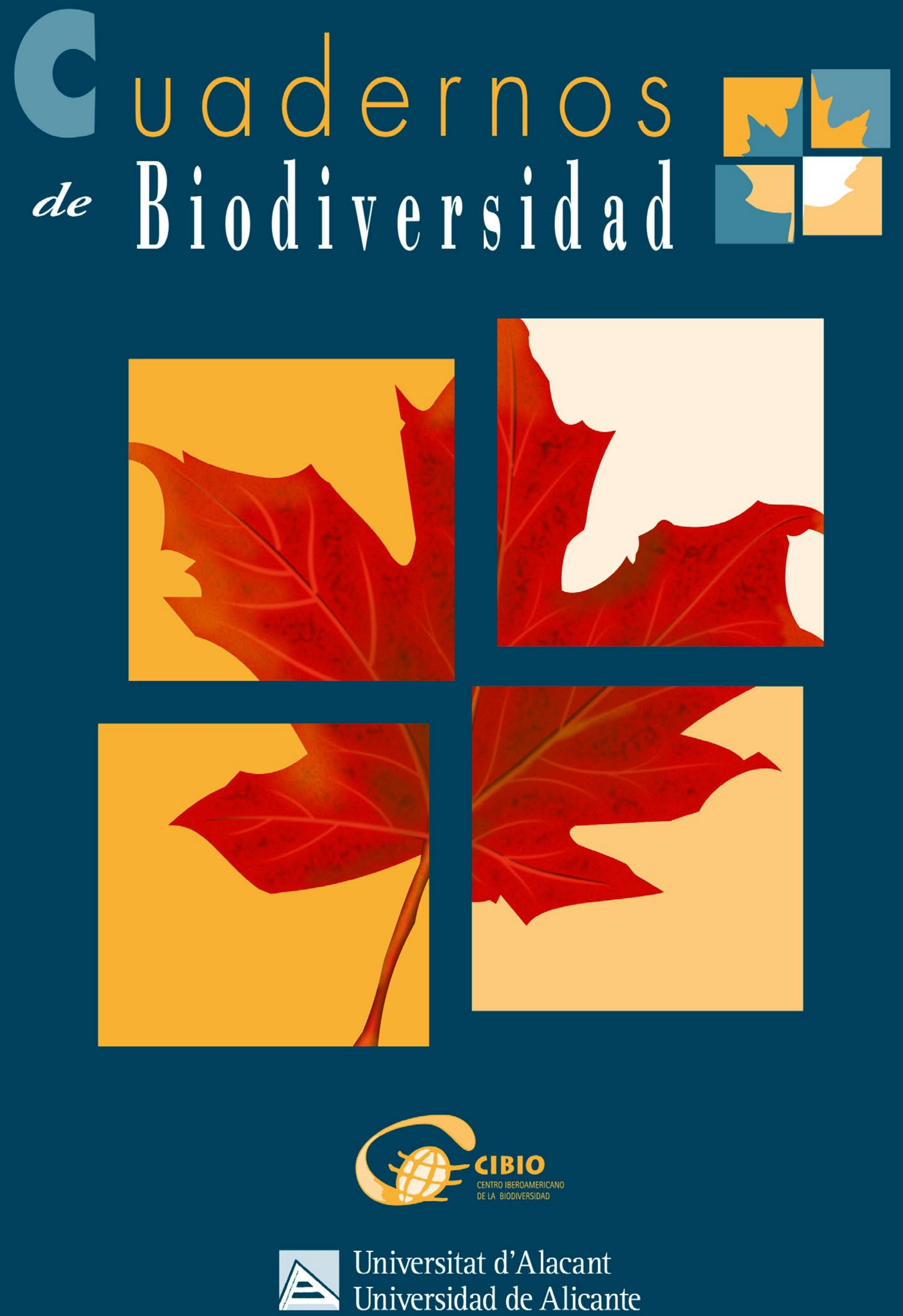


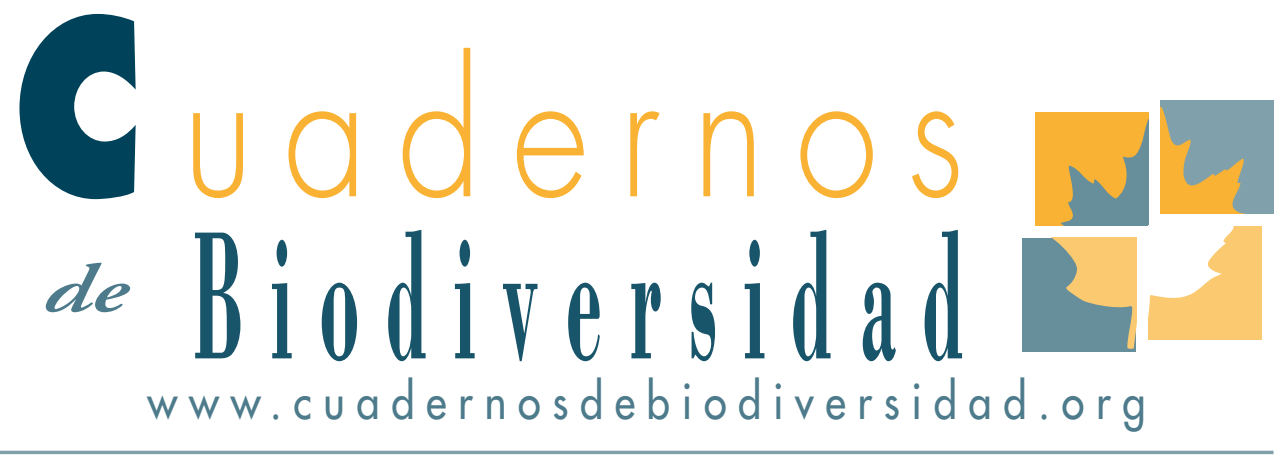

\section{Manglares, desarrollo turístico y cambio climático en Playa del Carmen, corazón del caribe mexicano}

\section{Mangroves, tourism development and climate change in Playa del Carmen, heart of the mexican carribbean}

\section{J. D. Ruiz-Ramírez ${ }^{i *}$, G. D. López Montejo², R. J. Cabrera Guillermo $^{2}$}

i Departamento de Ciencias, División de Ciencias e Ingenierías, Universidad de Quintana Roo, C.P. 77019, Chetumal, Quintana Roo, México.

2 Centro de Información Geográfica, División de Ciencias e Ingenierías, Universidad de Quintana Roo, C.P. 770 19, Chetumal, Quintana Roo, MÉxico.

*jenifer@uqroo.edu.mx

\section{RESUMEN}

Los manglares sostienen la biodiversidad de los ecosistemas costeros intertropicales del planeta; pero son amenazados constantemente por el desarrollo acelerado antrópico y los impactos del Cambio Climático (CC). En México, a pesar de estar protegidos por la ley, se requieren más estudios a escala local, por lo que se eligió la ciudad de Playa del Carmen, con la mayor tasa de crecimiento en México y América Latina. Se cartografiaron las superficies de manglar, utilizando una imagen de satélite QuickBird, se describe el desarrollo turístico de la ciudad y se señalan los principales impactos ante el CC, por huracanes y aumento del nivel del mar, obtenidos de datos mareográficos y altimétricos (TOPEX/Poseidón, Jason-1 y OSTM/Jason-2). Playa del Carmen cuenta con una extensión de 182 ha de manglares; su desarrollo acelerado lo ha convertido en un destino turístico de clase mundial; y sus tasas de aumento del nivel del mar (local y regional), son menores a la tasa mundial. Se concluye que el impacto temporal de los huracanes será mayor a medio plazo que el impacto paulatino del nivel medio del mar 
a largo plazo. Finalmente, se propone un "Área de Protección Especial” para salvaguardar el reducido ecosistema de manglar.

Palabras clave: aumento del nivel del mar, huracanes, Riviera Maya.

\section{ABSTRACT}

Mangroves sustain the biodiversity of the planet's intertropical coastal ecosystems; but they are constantly threatened by anthropogenic accelerated development and the impacts of Climate Change (CC). In Mexico, despite being protected by law, more studies are required on a local scale, which is why the city of Playa del Carmen was chosen, with the highest growth rate in Mexico and Latin America. The mangrove surfaces were mapped, using a QuickBird satellite image, the coastal development of the city is described and the main impacts due the $\mathrm{CC}$ are indicated, by hurricanes and sea level rise, obtained from tide and altimetric data (TOPEX / Poseidon, Jason-1 and OSTM / Jason-2). Playa del Carmen has an extension of 182 ha of mangroves; its accelerated development has turned it into a worldclass tourist destination; and their rates of sea level rise (local and regional), are lower than the world rate. It is concluded that the temporary impact of hurricanes will be greater in the medium term than the gradual impact of the long-term mean sea level. Finally, a "Special Protection Area" is proposed to safeguard the reduced mangrove ecosystem.

Key words: sea level rise, hurricanes, Mayan Riviera.

\section{INTRODUCCIÓN}

Los manglares constituyen un importante recurso forestal en toda la banda intertropical del planeta. Sostienen la biodiversidad de los ecosistemas costeros tropicales, en los humedales intermareales y áreas de influencia tierra adentro. Los ecosistemas de manglar cuentan con características específicas de estructura y función (ribereño, de borde, de cuenca, etc.). El paisaje costero donde se desarrollan los manglares, es vulnerable a los fenómenos naturales, como deslizamientos de tierra, subsidencia, huracanes, aumento del nivel del mar; pero las acciones antrópicas acrecientan las amenazas y riesgos naturales sobre los ecosistemas y esto ha sido más catastrófico que el propio cambio climático (Yañez-Arancibia et al., 1998).

Los manglares del mundo están desapareciendo rápidamente, con una pérdida anual de $1-2 \%$, debido principalmente a la urbanización, la construcción de carreteras, la contaminación, el turismo y la acuacultura (Hirales-Cota et al., 2010). México ocupa el quinto lugar con mayor extensión de manglares a nivel mundial, lo que representa un 5\% de cobertura total mundial (CONABIO, 2017a; García López, 2018; Valderrama-Landeros et al., 2017); éstos se encuentran protegidos bajo la Norma Oficial Mexicana NOM-022-SEMARNAT-2003, que establece las especificaciones para la preservación, conservación, aprovechamiento sustentable y restauración de los humedales costeros en zonas de manglar (CONACYT, 2016; Pérez, 2017; SEMARNAT, 2003). Las cuatro especies Laguncularia racemosa (L.) C.F. Gaertn (blanco), Conocarpus erecta L. (botoncillo), Avicennia germinans (L.) L. (negro) y Rhizophora mangle L. (1753) (rojo) están consideradas bajo protección ambiental en la Norma Oficial Mexicana NOM-059-SEMARNAT-2010, con el estatus de especies amenazadas (SEMARNAT, 2010). El Inventario Forestal Nacional de los años 70's indicaba una superficie de $1,5 \times 10^{6}$ ha, pero el Inventario Forestal de Gran Visión de 1992 señaló que sólo quedaban poco más de $500 \times 10^{3}$ ha, reflejando una tasa de deforestación de 60\% en 20 años (Yañez-Arancibia et al., 1998). Sin embargo, la Comisión Nacional para el Conocimiento y Uso de la Biodiversidad (CONABIO), ha llevado a cabo un Sistema de Monitoreo de los Manglares de México, y el inventario para el año 2015 registra 775555 ha de manglar, esto significa que desde 1970/1980 hasta 2010 predominaron las pérdidas de hectáreas de manglar, mientras que entre 2010 a 2015, prevalecieron las ganancias, resultado de la recuperación y la colonización de nuevas áreas (CONABIO, 2017a; Valderrama-Landeros et al., 2017). 
A pesar de estar protegidos por la ley, los manglares han estado amenazados por el desarrollo (urbano) acelerado en las zonas costeras en las últimas décadas (CONABIO, 2017a y 2017b; García López, 2018; Hirales-Cota et al., 2010; Valderrama-Landeros et al., 2017). Además, sufrirán los impactos del aumento del nivel del mar y cualquier cambio en la frecuencia e intensidad de futuras tormentas y huracanes, como consecuencia del cambio climático global (Ponce-Vélez et al., 2011; Shepard et al., 2012). Otro sistema que es sensible a los cambios ambientales es la duna costera; ha sido impactada y reducida en su extensión a nivel mundial y regional, y se considera que cerca del $70 \%$ de las playas del mundo se están erosionando, es decir están perdiendo sedimentos, ocasionando un alto costo para la población y la sociedad en general (Flores Verdugo, et al., 2010; Scott, et al., 2012).

En Quintana Roo (el único estado del país que es bañado por el Mar Caribe), se encuentran registradas 11 Áreas Naturales Protegidas (ANP's), con una superficie total de 312864,672 ha, de las cuales $90,91 \%$ son de competencia estatal y $9,09 \%$ son de competencia municipal, en ellas se protegen diferentes ecosistemas, incluida la vegetación de manglar (CONACYT, 2016).

Este trabajo se enfoca en la ciudad costera de Playa del Carmen, corazón de la Riviera Maya en el Caribe mexicano. Es un atractivo destino turístico de sol y playa, que por su rápido desarrollo ha alcanzado la posición de la ciudad con mayor tasa de crecimiento en México y América Latina (6,8\% anual, pero alcanzó el $32 \%$ en el periodo 2005-2015), principalmente por las actividades económicas destinadas al turismo (INEGI, 2015). Su oferta hotelera es mayor a la de Cancún, al tener 44000 habitaciones (10000 más que Cancún), convirtiéndolo en el principal destino de turistas provenientes de Estados Unidos, Canadá y Europa. Su derrama económica anual, supera los USD\$ 8000 millones (APIQROO, 2018; Góchez, 2015; SEDETUR, 2017).

Por lo que el objetivo fue clasificar las principales coberturas para determinar el área de manglar que posee actualmente, describir el estado actual del desarrollo turístico de Playa del Carmen y señalar las amenazas (erosión de playa y huracanes) como posibles impactos del cambio climático en esta zona.

\section{MATERIALES Y MÉTODOS}

\section{Área de estudio}

La ciudad costera de Playa del Carmen se encuentra localizada en el centro del estado de Quintana Roo, en el municipio de Solidaridad, en las coordenadas $20^{\circ} 38^{\prime} 00^{\prime \prime} \mathrm{N}$ y $87^{\circ} 05^{\prime} 00^{\prime \prime} \mathrm{O}$. Se encuentra sobre una planicie de origen tectónico, siendo las máximas elevaciones inferiores a los 25 metros sobre el nivel del mar, que disminuyen hacia la zona de la costa. En su suelo predomina la roca caliza, sumamente permeable, que no permite la existencia de corrientes de aguas superficiales; por el contrario, cuenta con innumerables cenotes. Los cenotes en su mayoría son ensanchamientos de complejas redes fluviales subterráneas, que en ocasiones se conectan al mar. En éstos, el agua marina, más densa que la dulce, puede penetrar por el fondo del sistema freático, permitiendo que a determinada profundidad el agua pase de dulce a salada (Municipio de Solidaridad, 2016a). El clima del municipio es cálido subhúmedo con lluvias en el verano, la temperatura media anual es de $26^{\circ} \mathrm{C}$ y los vientos predominantes son los del sureste. El clima se ve afectado por las tormentas o huracanes, donde la temporada se extiende del 1 de junio al 30 de noviembre de cada año (Municipio de Solidaridad, 2016a; NOAA, 2016).

\section{Vegetación}

La vegetación se conforma de selva mediana subperennifolia y subcaducifolia, y selva baja subperennifolia, que son particularmente valiosas para la explotación forestal debido a la presencia de maderas preciosas como la caoba y el cedro. En zonas próximas a las áreas inundables y al mar predominan las comunidades de manglares, con las especies $R$. mangle (rojo) y L. racemosa (blanco), además de $A$. germinans (negro) y $C$. erectus (botoncillo), aunque la superficie que ocupan es relativamente pequeña (Yañez-Arancibia et al., 1998; Municipio de Solidaridad, 2016a). Las comunidades de las dunas son consideradas halófitas, debido a que los suelos 
poseen un alto contenido de sales solubles, tienen una distribución heterogénea a lo largo de la costa, con una mezcla de especies herbáceas, matorrales arbustivos o especies arbóreas, algunos ejemplos son Sesuvium portulacastrum (verdolaga de playa) e Ipomea pes-caprae (bejuco de playa) (CONABIO, 2018).

\section{Clasificación de las coberturas}

Para clasificar el mapa de la ciudad de Playa del Carmen y determinar sus coberturas, se seleccionó una imagen del satélite QuickBird de fecha 2015, la cual posee una alta resolución espacial (tamaño de pixel de $0,5 \mathrm{~m}$ ). Se seleccionó este sensor porque la superficie de interés corresponde a un área urbana con diferentes tipos de coberturas en áreas relativamente pequeñas (la escala de la clasificación es 1:5000). El proceso de clasificación a partir de la imagen seleccionada fue la fotointerpretación y digitalización de los polígonos correspondientes a las coberturas: a) Franja de arena, corresponde a las dunas y playas en la costa; b) Humedal, corresponde a las zonas inundables con mezcla de agua dulce y salada; c) Manglar, corresponde al área cubierta con este tipo de vegetación; d) Área urbana, incluye a las zonas de manzanas, vialidades, infraestructura urbana y turística de la ciudad. Para definir la clase Manglar se utilizó, como referencia, el mapa digital "Distribución de los manglares en México" generado por la Comisión Nacional para la Biodiversidad (CONABIO, 2017b), para comparar los polígonos obtenidos de la digitalización y aumentar la exactitud del resultado. Posterior a la clasificación se estimaron las superficies de cada una de las clases.

\section{Desarrollo costero}

Se consultaron fuentes oficiales para reflejar el proceso de crecimiento urbano y turístico de la ciudad de Playa del Carmen, desde que era un territorio ocupado por los mayas, seguido por la conquista española, la expansión en la guerra de castas, la actividad chiclera de la época y finalmente, como destino turístico.

\section{RESULTADOS Y DISCUSIÓN}

La ciudad de Playa del Carmen cuenta con una extensión de 182,080 ha de manglar, que se distribuye principalmente en su franja costera, este ecosistema convive y se mezcla con el desarrollo urbano y turístico de la ciudad (Fig. 1).

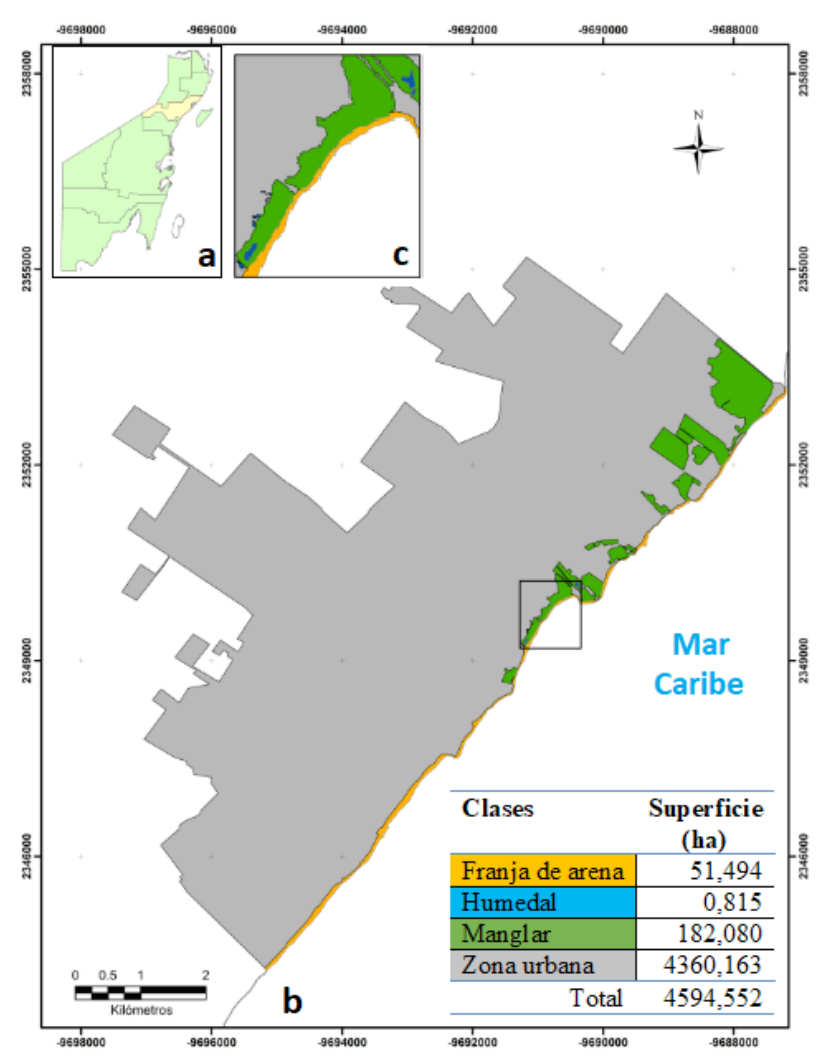

Fig. 1. Playa del Carmen, Quintana Roo. a) Ubicación del municipio Solidaridad en el Estado de Quintana Roo, b) Clasificación de los principales componentes de la ciudad y c) Detalle del área de Humedales. Elaboración propia.

Esta extensión de manglar no se encuentra protegida, es decir, el municipio de Solidaridad, donde se encuentra ubicada Playa del Carmen, carece de un ANP. 


\section{Desarrollo costero}

El territorio donde se ubica Playa del Carmen, pertenecía en su mayor parte al cacicazgo de Ecab, que comprendía las localidades de Tulum, Cobá, XamanHá (actualmente Playa del Carmen), Tancah y Polé. La región fue conquistada en 1526 por Francisco de Montejo quien fundó la villa de Salamanca (actualmente Xel-Ha). En las crónicas de los españoles se describe a Tulum como una ciudad tan grande como Sevilla. Durante la época colonial la región permaneció casi deshabitada y no fue hasta el siglo XX cuando se fundaron campamentos para la explotación del chicle y la madera (Municipio de Solidaridad, 2016b).

La historia moderna de Playa del Carmen empezó con el arribo de las primeras familias provenientes de la Península de Yucatán, obligadas por las contingencias de la guerra de castas (1847-1901) en busca de tierras menos conflictivas donde establecerse. Otra versión señala que, al ser contratados como chicleros, algunos desertaron y decidieron trabajar como pescadores, para quedarse a vivir en esas tierras. Comienza entonces un lento desarrollo, propiciado por el crecimiento de las familias (Municipio de Solidaridad, 2016b).

A partir de los años 60's se emprende un rápido y progresivo crecimiento de la población, y Playa del Carmen deja de ser un lugar de paso hacia Cozumel y principia su actividad económica. La población se distribuía principalmente entre las localidades de Playa del Carmen, Tulum, Puerto Aventuras, Cobá y otras 14 localidades litorales con población mayor de 100 habitantes (Municipio de Solidaridad, 2016b).

A finales de 1992 contaba con 10594 habitantes y tenía en operación 500 nuevos cuartos hoteleros. Poseía además una zona indígena, de enorme riqueza cultural y arqueológica, cuyo centro era Cobá. Para entonces, la población de Playa del Carmen manifestó abiertamente su inquietud para la creación de un nuevo municipio libre y se decreta el 28 de julio de 1993, el Municipio de Solidaridad. En tan sólo 20 años, Playa del Carmen pasó de ser una villa de pescadores a un destino turístico de interés mundial (Fig. 2) (INEGI, 2016a; INEGI, 2016b; Municipio de Solidaridad, 2016b).

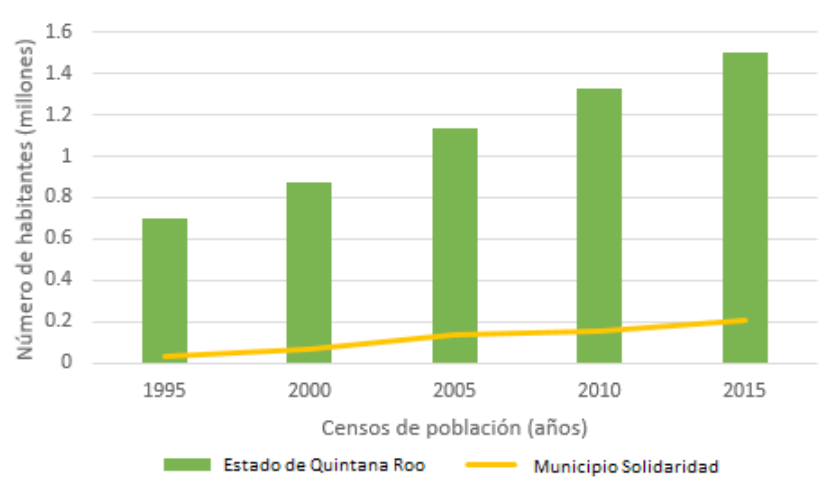

Figura 2. Crecimiento de la población del Municipio de Solidaridad desde su decreto de creación (Datos de INEGI, 2016a, 2016b).

\section{Cambio climático}

Las principales amenazas a las que está expuesta la zona costera de la península de Yucatán, son los eventos extremos naturales, huracanes y "nortes", los disturbios antropogénicos puntuales como los asentamientos humanos, que incluyen la construcción de hoteles y casas en la zona de dunas y manglares, y el cambio climático global (Liceaga-Correa et al., 2010).

\section{- Huracanes}

La zona del Caribe mexicano está afectada por tormentas y huracanes, principalmente los que se originan en la costa occidental de África, cerca de las islas de Cabo Verde (Guido-Aldana et al., 2009). Cada año, la temporada de huracanes para el Atlántico inicia el 1 de junio y termina el 30 de noviembre (NOAA, 2016).

El Atlas Climatológico de Ciclones Tropicales en México, señala que la Península de Yucatán está expuesta a un promedio de más de 20 ciclones tropicales en 150 años (Rosengaus Moshinsky et al., 2014). La NOAA señala que la cuenca tropical de ciclones del Atlántico, formada por el Océano Atlántico, Mar Caribe y Golfo de México, ha experimentado desde 1995 un incremento en la frecuencia de huracanes más intensos con categorías de tres a cinco, siendo esta última, la máxima en la escala Saffir-Simpson (Liceaga-Correa, et al., 2010). El IPCC señala que desde la década de 1970 ha habido un aumento en la frecuencia e intensidad de los 
ciclones tropicales en la región del Atlántico Norte, pero esto no ocurre a nivel mundial, al compararlos con la actividad ciclónica tropical durante el siglo XX (IPCC, 2014; Knutson, et al., 2010; Wong, et al., 2014).

El huracán Wilma (categoría 4 en la escala SaffirSimpson) en 2005, fue el huracán más intenso jamás registrado en el Atlántico y el que más ha afectado el litoral caribeño mexicano en los últimos 70 ańos; estuvo estacionado por 48 horas en las costas de Cancún, Cozumel y Playa del Carmen, y registró vientos sostenidos de $295 \mathrm{~km} / \mathrm{h}$ y rachas de 350 $\mathrm{km} / \mathrm{h}$, alcanzando una presión mínima central de $882 \mathrm{mb}$ (Pasch et al., 2006; Martell-Dubois et al., 2012).

Este huracán afectó a 62000 turistas que se encontraban en Cancún y la Riviera Maya (Verdayes Ortiz, 2013; Miranda Jácome, 2014). Según la Asociación Mexicana de Instituciones de Seguro (AMIS), fue el desastre natural más caro que haya tenido México, ya que su impacto en las costas de Quintana Roo, ocasionaron daños equivalentes a USD\$ 10000000000 (Vázquez Botello, 2008), de los cuales USD\$ 350000000 fueron los daños calculados sobre la infraestructura hotelera de Cancún (CEPAL, 2006).

La erosión en Cancún, provocada por Wilma, eliminó más del 70\% de las playas. Las autoridades decidieron ejecutar un programa de vertimiento de
$2700000 \mathrm{~m} 3$ de arena a principios de 2006, para regenerar la playa de manera artificial, costándole a la federación USD\$23400000, siendo efectiva por 3 años. La restauración garantizaba el arribo de turistas y la activación económica (Tabla 1) (Guido Aldana et al., 2009; Martell-Dubois et al., 2012).

Posteriormente, el huracán Dean en 2007 (categoría 5 en la escala Saffir-Simpson, con vientos sostenidos de $260 \mathrm{~km} / \mathrm{h}$ y con una presión mínima central de $905 \mathrm{mb}$ ), afectó a Mahahual, que fue la única ciudad que experimentó toda la fuerza del huracán. Los destinos turísticos de Cancún y Cozumel reportaron daños relativamente leves. El Puerto Costa Maya, el segundo puerto de cruceros más importante del Estado y del país, sufrió graves daños, por arriba de USD\$ 15000000 y estuvo cerrado por un ańo y dos meses. Este muelle recibe anualmente 1000000 de turistas en este destino, en aproximadamente 356 cruceros. Este huracán causó pérdidas económicas por USD\$ 100000000000 en México y en el vecino país de Belice se reportaron USD\$ 100000000 en daños (Franklin, 2008; Torres Alvarado \& Calva Benítez, 2011).

Dean provocó una nueva pérdida de arena, de 30 $m$ de ancho de playa (Guido Aldana et al., 2009; Martell-Dubois et al., 2012). Los empresarios e inversionistas del Caribe mexicano solicitaron el vertimiento de arena para Cancún, y que además se incluyera la Riviera Maya, Isla Mujeres y Cozumel. En 2009, la Secretaría de Turismo Federal autorizó la reposición de 16,800 km de playas para Cancún,

Tabla 1. Erosión en las playas de Cancún y costo de mantenimiento

\begin{tabular}{|c|c|c|c|c|}
\hline Evento & $\begin{array}{c}\text { Erosión de playa } \\
\text { (Ancho) }\end{array}$ & $\begin{array}{c}\text { Recuperación } \\
\text { A (ancho) } \\
\text { L (longitud) }\end{array}$ & $\begin{array}{l}\text { Vertimiento de } \\
\text { arena }\left(\mathbf{m}^{3}\right)\end{array}$ & Costo (USD) ${ }^{1}$ \\
\hline Huracán Wilma $(2005)^{*}$ & $70 \mathrm{~m}$ & $70 \mathrm{~m} \mathrm{~A}$ & 2700000 & 23400000 \\
\hline Sin huracán $(2009)^{* *}$ & - & $16,8 \mathrm{~km} \mathrm{~L}$ & 6100000 & 71400000 \\
\hline Sin huracán $(2013)^{* *}$ & $10-15 \mathrm{~m}$ & $16,0 \mathrm{~km} \mathrm{~L}$ & - & 15400000 \\
\hline & & & Total & $\$ 110200000$ \\
\hline
\end{tabular}

1 Tasa de cambio de \$USD acorde al año referido (Banxico, 2017)

* Sólo Cancún

** Cancún, Playa del Carmen y Cozumel

Elaboración propia con datos de Guido Aldana et al., 2009; Martell-Dubois et al., 2012; Moguel, 2013. 
Playa del Carmen y Cozumel. Se requirió extraer y bombear más de $6100000 \mathrm{~m} 3$ de material arenoso de los bancos de arena en Isla Mujeres y Cozumel, con una inversión cercana a los USD\$ 71400000 (3 veces mayor a la del 2005); la mitad de esa cantidad la aportó el gobierno federal y el resto fue proporcionado por el gobierno de Quintana Roo y hoteleros y empresarios de los destinos turísticos (Tabla 1) (Guido Aldana et al., 2009; Sánchez, 2009).

Por último, el huracán Ernesto en 2012 (categoría 2 en la escala Saffir-Simpson y con una presión mínima central de $975 \mathrm{mb}$ ), volvió a afectar el litoral mexicano. Esta vez, 1500 turistas fueron evacuados de centros turísticos en el Estado y los daños fueron estimados en USD\$174000000, de los cuales, USD\$ 76400000 fueron en pérdidas agrícolas y daños en carreteras (Brown, 2013).

En 2013, después de Ernesto, la recuperación de playas de Cancún abarcó 16,000km distribuidos en Cancún, Cozumel y Playa del Carmen, con un costo de USD\$ 15400000 (Tabla 1) (Moguel, 2013).

El impacto temporal y permanente de los huracanes en el Caribe mexicano, debido principalmente a la fuerza de los vientos, las mareas de tormenta y las inundaciones, destruyen y paralizan la actividad económica principal del Estado, en menos de una década, tres huracanes de distinta categoría y trayectoria, han causado pérdidas significativas en la reconstrucción de infraestructura turística y urbana, y tan sólo para la recuperación de playas se han invertido USD \$110200000.

Entre las causas que intensifican los efectos de los huracanes, se incluyen la degradación de manglares y humedales, la erosión de playas y pérdida de suelo (Guido Aldana et al., 2009). Señalar el impacto de los huracanes en la zona costera merece atención especial, por su contribución a la economía nacional, ya que la entidad aporta el $34,1 \%$ del total de divisas que ingresan al país, por medio del sector turismo (SEDETUR, 2018).

\section{- Aumento del nivel del mar}

El aumento del nivel del mar en las costas (SLR por sus siglas en Inglés Sea Level Rise) se reconoce como uno de los resultados más directos del calentamiento global. En los últimos 100 años, se ha incrementado en promedio de 1 a $2 \mathrm{~mm}$ por año y se anticipa que esas tasas se acelerarán en las próximas décadas (Hernández Arana, 2007). Para la región del Mar Caribe, se encontró una tasa promedio de 2,000 mm ańo-1, con datos obtenidos de la serie de tiempo 1993-2013 de los altímetros TOPEX/Poseidón, Jason-1 y OSTM/Jason-2 (Ruiz-Ramírez et al., 2014). Sin embargo, esta tendencia va en aumento pues al analizar la serie de tiempo de 1993-2014 la tasa registraba 2,300 $\mathrm{mm}$ año-1 y la serie de tiempo de 1993-2015 registró una tasa de 2,800 mm año-1 (Tabla 2).

Tabla 2. Tendencia del aumento del nivel del mar para la región del Mar Caribe

\begin{tabular}{l|c|c|} 
Región & Período & $\begin{array}{c}\text { Tasa de SLR (mm } \\
\left.\text { ańo }^{-1}\right) \text { obtenido de } \\
\text { altímetros }\end{array}$ \\
\hline Mar Caribe & $1993-2013^{*}$ & 2,000 \\
& $1993-2014$ & 2,300 \\
$1993-2015$ & 2,800
\end{tabular}

*Este dato fue reportado por Ruiz-Ramírez et al., 2014. Elaboración propia

A ese ritmo, pronto se alcanzará la tasa de aumento del nivel del mar mundial de $3,400 \mathrm{~mm}$ año-1, registrada por la Universidad de Colorado y las agencias AVISO y NASA (Universidad de Colorado, 2017). En contraste, a nivel local, los mareógrafos instalados en el Caribe mexicano, reportan una tasa de aumento de nivel del mar de 1,600 mm año-1, menor que la tasa regional y la mitad de la tendencia mundial (Tabla 3) (Ruiz-Ramírez et al., 2014). 
Tabla 3. Comparación de la tendencia de SLR a distintas escalas (elaboración propia).

\begin{tabular}{l|l|l} 
Escala & $\begin{array}{l}\text { Tendencia (mm } \\
\text { año-1) }\end{array}$ & Instrumento \\
\hline $\begin{array}{l}\text { Local (Caribe } \\
\text { mexicano) }\end{array}$ & 1,6 & Mareógrafo \\
\hline $\begin{array}{l}\text { Regional (Mar } \\
\text { Caribe) }\end{array}$ & 2,8 & Altímetro \\
\hline Mundial & 3,4 & $\begin{array}{l}\text { Mareógrafo y } \\
\text { altímetro }\end{array}$
\end{tabular}

Las tasas de incremento de SLR a diferentes escalas resultaron positivas, y aunque aparentemente el aumento en el nivel del mar mundial no representa un peligro para las costas del Caribe mexicano, porque las tendencias local y regional son menores, es necesario señalar que la vulnerabilidad costera desde el punto de vista de exposición, aumenta por la topografía relativamente plana de su relieve y su escasa altura sobre el nivel del mar.

Siendo Playa del Carmen la segunda localidad más poblada del estado (209634 habitantes), después de Cancún, posee un ritmo de crecimiento poblacional mayor. La dinámica es tal, que las grandes marcas y plazas han encontrado un nicho de mercado, que siguen desarrollando, por lo que su oferta hotelera es mayor a la de Cancún (44000 habitaciones), esto lo convierte en el principal destino de turistas provenientes de Estados Unidos, Canadá y Europa (Góchez, 2015).

$\mathrm{Al}$ ser un destino de clase mundial, es un sitio que extranjeros y nacionales eligen para establecerse. El costo promedio por metro cuadrado de construcción en la ciudad es de USD $\$ 4000$ con base al precio de venta con frente de playa (Tabla 4). El Modelo Digital de Elevación (MDE) de la ciudad de Playa del Carmen revela una altitud promedio de 6,700 msnm. Al combinar el área turística y urbana con su MDE, se analizaron diferentes escenarios de inundación de 1 a $3 \mathrm{~m}$ por el nivel del mar, y se calcularon las superficies afectadas con valores actuales de mercado, obteniéndose que a) el escenario a $1 \mathrm{~m}$ de elevación, afectaría una superficie de $19281 \mathrm{~m} 2$, con un valor estimado de USD\$ 77000000; b) el escenario a 2 m de elevación, afectaría una superficie de $64649 \mathrm{~m} 2$, con un valor estimado de USD\$ 258000000; y c) el escenario a 3 m de elevación afectaría una superficie de $90895 \mathrm{~m} 2$ con un valor estimado de USD\$363000000 (Tabla 4).

Tabla 4. Superficie de afectación de la ciudad de Playa del Carmen de acuerdo al Modelo Digital de Elevación y costo estimado por $\mathrm{m} 2$

\begin{tabular}{|c|c|c|c|c|c|}
\hline $\begin{array}{l}\text { Costo } \\
\text { por } \mathrm{m}^{2} \mathrm{de} \\
\text { construcción }\end{array}$ & \multicolumn{3}{|c|}{$\begin{array}{l}\text { Superficie de infraestructura afectada }\left(\mathrm{m}^{2}\right) / \mathrm{km} \text { de } \\
\text { playa y su valor en USD\$ y total del sitio en los } \\
\text { escenarios de elevación del mar }\end{array}$} & $\begin{array}{l}\text { Longitud del } \\
\text { tramo de costa } \\
\text { analizado }\end{array}$ & $\begin{array}{l}\text { Elevación media } \\
\text { (msnm) del } \\
\text { MDE LiDAR }\end{array}$ \\
\hline \multirow{3}{*}{$\$ 4000$} & $1 \mathrm{~m}$ & $2 \mathrm{~m}$ & $3 \mathrm{~m}$ & \multirow{3}{*}{5,700} & \multirow{3}{*}{$6,700^{*}$} \\
\hline & $19281 \mathrm{~m}^{2}$ & $64649 \mathrm{~m}^{2}$ & $90895 \mathrm{~m}^{2}$ & & \\
\hline & $\$ 77124000$ & $\$ 258596000$ & $\$ 363580000$ & & \\
\hline
\end{tabular}

Nota: El valor en dólares se calculó en base al promedio del $\mathrm{m} 2$ de venta con frente de playa para la ciudad, según la AMPI (Asociación Mexicana de Profesionales Inmobiliarios).

* Partiendo de la línea costera hacia $5 \mathrm{~km}$ tierra adentro

(elaboración propia) 
Estudios previos en la zona (Ortiz Pérez \& Méndez Linares, 1999; Vázquez Botello, 2008; Blanchon et al., 2010; Flores Verdugo et al., 2010; Márquez García et al., 2010) revelan que aumentos de hasta $50 \mathrm{~cm}$ a $1 \mathrm{~m}$ pueden ser devastadores, especialmente para la infraestructura turística que ocupa áreas susceptibles de inundación, provocando erosión de las playas por el oleaje y ocasionando un fuerte impacto a la economía estatal.

Para la Riviera Maya, ya se ha estimado que un incremento del nivel del mar de $50 \mathrm{~cm}$, provocaría la desaparición del $20 \%$ de las playas; pero si se eleva a $1 \mathrm{~m}$, el $90 \%$ de las playas actuales desaparecerán; sin embargo, esa evaluación no considera los posibles cambios que existieran por huracanes, disminución de transporte litoral o la construcción de alguna obra civil, entre otros procesos o actividades (Márquez García et al., 2010). En este trabajo, el escenario a $1 \mathrm{~m}$ de SLR, afectaría una superficie de $19281 \mathrm{~m} 2$, con un valor estimado de USD\$77000000, pero este valor no considera niveles o números de pisos de edificios, su mobiliario y los servicios básicos (agua potable, luz eléctrica, televisión por cable e internet) de cada inmueble.

A estas cantidades, se le puede atribuir un aumento de aproximadamente $30 \%$ en daños y perjuicios debido al calentamiento global; pero este impacto es de un valor extremadamente pequeño, ya que cada 10-15 años ocurre una duplicación de los dańos debido al aumento de la población combinado con el aumento de la infraestructura y actividades a lo largo de las costas (Caetano et al., 2010).

En relación a los manglares, los impactos del aumento del nivel del mar sobre éstos, dependerán de la tasa del incremento en relación con la acreción vertical y el espacio para la migración horizontal, ya que provocaría un desplazamiento tierra adentro de los manglares que puede estar limitadas por el desarrollo urbano y el turismo en zonas costeras (Ponce-Vélez et al., 2011). El estudio revela que la extensión del manglar-humedal ocupa apenas un
$3,9 \%$, en comparación con un $95 \%$ de extensión urbana-turística, por lo que la migración horizontal se descarta y la acreción vertical se desconoce, por falta de estudios en la zona.

Se considera que la distribución preferencial de una especie de manglar, su expansión o compresión del área, va a depender de las características topográficas tierra adentro, del tipo de costa en función de su origen geomorfológico y la combinación de las características climáticas regionales (Flores Verdugo et al., 2011). Para las costas del Caribe, se presentaría una pérdida neta significativa en extensión del manglar de $2954 \mathrm{~km} 2$ que equivale al $74 \%$ con un escenario de menos de un metro de SLR para finales de este siglo; de manera particular por su escaso nivel de mareas $(<50 \mathrm{~cm})$, así como el efecto del aumento en la frecuencia de los huracanes de mayor categoría y el deterioro de su barrera natural (el arrecife de coral) (Flores Verdugo et al., 2010).

Ante esos escenarios, los ecosistemas de manglar al perder extensión, tendrían una respuesta selectiva de las especies más tolerantes a rangos de salinidad y temperatura. En la zona, el ecosistema arrecifal ya se encuentra presionado, paulatinamente ya no podrán proteger las costas, ni suministrar sedimentos para las playas; $y$ ambos son primordiales para proteger las costas ante el embate de los huracanes, que ya son más frecuentes y más intensos en la cuenca del Caribe. Las playas y dunas, al igual que los manglares, podrían migrar, pero cada vez los cambios espaciales y temporales son más restringidos y más rápidos, lo que no le proporciona el tiempo necesario a estos ecosistemas de adaptarse (Flores Verdugo, et al., 2010). Además, la erosión se acentúa por causas antropogénicas debido a la construcción de infraestructura (urbana y turística) y a la modificación de los volúmenes de descarga por aporte de sedimentos fluviales, situación que incrementa el efecto erosivo de las corrientes marinas y litorales, y el resultado es una fuerte erosión costera y pérdida de grandes extensiones de playa (Bolongaro Crevenna Recaséns, et al., 2010). 


\section{CONCLUSIONES}

Playa del Carmen es la segunda ciudad más poblada del Caribe mexicano, pero con una tasa de crecimiento superior a la de México y América Latina. La ciudad atrae la mayor inversión en el ramo turístico, incrementando la dinámica del desarrollo costero. Esto ha repercutido en la reducción de las áreas ocupadas por el ecosistema de manglar, por lo que se propone un "Área de Protección Especial", que ya está planteado en el Programa de Desarrollo Urbano de Playa del Carmen (2010), para que este ecosistema sobreviva, perdure y conviva con los habitantes nacionales y extranjeros, con el propósito de que exista un ANP en el municipio de Solidaridad, siga brindando sus servicios ambientales, y éstos se difundan con fines educativos y de sensibilización a la población.

La ciudad está expuesta principalmente a dos impactos relacionados con el cambio climático, los huracanes y el aumento del nivel del mar. Los huracanes han provocado dańos considerables a la infraestructura urbana y turística, y en sus playas, las intervenciones de vertimientos de arena se están convirtiendo en una constante para mantener atractivas las actividades económicas. Por otro lado, el aumento del nivel del mar, aunque todavía es menor la tendencia local y regional a la tasa mundial, la baja elevación del terreno en la costa evidenciada en el MDE revela que bajo diferentes escenarios de inundación, la afectación de la superficie y sus habitantes, por elevación del nivel medio del mar, impactarán los desarrollos turísticos, con efectos negativos sobre la economía estatal, debido principalmente a la concentración de bienes y su valor actual en el mercado, que seguramente los propietarios y autoridades de los diferentes niveles de gobierno deberán evaluar de forma cuidadosa para no acrecentar los problemas de la zona, de ahí la importancia de mediciones más finas, monitoreo permanente y acciones pertinentes.

A corto plazo se vislumbra que el aumento en la frecuencia de tormentas y huracanes será más peligroso que el incremento del nivel del mar a largo plazo, pues la lección aprendida con Wilma paralizó las actividades económicas del turismo y afectó seriamente las necesidades de ingreso.
Algunas medidas de adaptación deberán enfocarse a las áreas no desarrolladas para limitar el desarrollo turístico y urbano en zonas de alto riesgo, la conservación y/o restauración del sistema de dunas y playas, protección de manglares, humedales y dunas costeras con un estatus legal, permitir el flujo hidrológico entre los ecosistemas de humedaleslagunas costeras-arrecifes, y prevenir la descarga de contaminantes, incluidas las aguas subterráneas por su conexión con el sistema de cenotes y recarga de acuíferos. Es necesario difundir información a usuarios sobre la importancia del mantenimiento de los servicios ambientales y el cuidado que el gobierno y la iniciativa privada han emprendido para enfrentar eficazmente los impactos directos e indirectos al cambio climático.

\section{AGRADECIMIENTOS}

La primera autora agradece la beca PRODEP otorgada a través de la Universidad de Quintana Roo (UQROO) para sus estudios de doctorado en el CINVESTAV-Unidad Mérida. A la Dra. Patricia Moreno Casasola y a un revisor anónimo por sus comentarios, que contribuyeron en gran medida al mejoramiento de este manuscrito.

\section{REFERENCIAS}

APIQROO (2018). Cozumel se coloca, por segundo año consecutivo, como líder en la industria de cruceros en México y Centroamérica. Administración Portuaria Integral de Quintana Roo S.A. de C.V. Gobierno del Estado de Quintana Roo 2016-2022. Publicado el 17 de septiembre de 2018. Recuperado en noviembre 30, 2018, disponible en: http://www.apiqroo.com. $\mathrm{mx} /$ cozumel-se-coloca-por-segundo-ano-consecutivocomo-lider-en-la-industria-de-cruceros-en-mexico-ysudamerica/

Banxico (2017). Mercado cambiario (tipos de cambio). Recuperado en junio 19, 2017, disponible en http:// www.banxico.org.mx/portal-mercado-cambiario/index. html 
Blanchon, P., Iglesias-Prieto, R., Jordán Dahlgren, E., \& Richards, S. (2010). Arrecifes de coral y cambio climático: vulnerabilidad de la zona costera del estado de Quintana Roo. En: Botello, A.V., VillanuevaFragoso, S., Gutiérrez, J. \& Rojas Galaviz, J.L. (eds.), Vulnerabilidad de las zonas costeras mexicanas ante el cambio climático. SEMARNAT-INE, UNAM-ICMyL, Universidad Autónoma de Campeche. Campeche, México: pp. 229-248.

Bolongaro Crevenna Recaséns, A., Márquez García, A.Z., Torres Rodríguez, V. \& García Vicario, A. (2010). Vulnerabilidad de sitios de anidación de tortugas marinas por efectos de erosión costera en el estado de Campeche. En: A.V. Botello, S. Villanueva-Fragoso, J. Gutiérrez, \& J.L. Rojas Galaviz (eds.). Vulnerabilidad de las zonas costeras mexicanas ante el cambio climático. SEMARNAT-INE, UNAM-ICMyL, Universidad Autónoma de Campeche. México: pp. 73-96.

Brown, D.P. (2013). Tropical Cyclone Report Hurricane Ernesto 1-10 August 2012. Recuperado en abril 17, 2017, disponible en http://www.nhc.noaa.gov/data/tcr/ AL052012_Ernesto.pdf

Caetano, E., Innocentini, V., Magańa, V., Martins, S. \& Méndez, B. (2010). Cambio climático y el aumento del nivel del mar. En: Botello, A.V., Villanueva-Fragoso, S., Gutiérrez, J. \& Rojas Galaviz, J.L. (eds.), Vulnerabilidad de las zonas costeras mexicanas ante el cambio climático. SEMARNAT-INE, UNAM-ICMyL, Universidad Autónoma de Campeche. Campeche, México. pp. 283304.

CEPAL (2006). Características e impacto socioeconómico de los huracanes Stan y Wilma en la República Mexicana en el 2005. Recuperado en junio 29, 2016, disponible en http://www.cepal.org/publicaciones/xml/0/27710/ L751-13.pdf

CONABIO (2017a). Actualización y exploración de los datos del sistema de monitoreo1970/1980-2015. Recuperado en noviembre 28, 2018, disponible en http://bioteca. biodiversidad.gob.mx/janium/Documentos/12889.pdf

CONABIO (2017b). Manglares de México: Extensión, distribución y monitoreo 2013. Recuperado en junio 20, 2017, disponible en http://www.biodiversidad.gob. mx/ecosistemas/manglares2013/manglaresMex_2013. html

CONABIO (2018). Biodiversidad mexicana, dunas costeras. Recuperado en noviembre 30, 2018, disponible en https://www.biodiversidad.gob.mx/ecosistemas/ dunasCosteras.html
CONACYT (2016). Áreas Naturales Protegidas del Estado de Quintana Roo. Recuperado en noviembre 23, 2016, disponible en http://conacyt.gob.mx/cibiogem/index. $\mathrm{php} / \mathrm{anpl} /$ quintana-roo

Flores Verdugo, F.J., Casasola, P., de la Lanza-Espino, G. \& Agraz Hernández, C. (2010). El manglar, otros humedales costeros y el cambio climático. En: Botello, A.V., Villanueva-Fragoso, S., Gutiérrez, J. \& Rojas Galaviz, J.L. (eds.), Vulnerabilidad de las zonas costeras mexicanas ante el cambio climático. SEMARNAT-INE, UNAM-ICMyL, Universidad Autónoma de Campeche. Campeche, México. pp. 165-188.

Flores Verdugo, F.J, Casasola, P., de la Lanza-Espino, G. \& Agraz, C. (2011). El manglar, otros humedales costeros y el cambio climático. En: Botello, A.V., VillanuevaFragoso, S., Gutiérrez, J. \& Rojas Galaviz, J.L. (eds.), Vulnerabilidad de las zonas costeras mexicanas ante el cambio climático. Universidad Autónoma MetropolitanaIztapalapa, UNAM-ICMyL, Universidad Autónoma de Campeche. Campeche, México. pp. 205-228.

Franklin, J.L. (2008). Tropical Cyclone Report Hurricane Dean 13-23 August 2007. Recuperado en mayo 11, 2017, disponible en http://www.nhc.noaa.gov/data/tcr/ AL042007_Dean.pdf

García López, T. (2018). Hacia una metodología para el diseño de programas de pago por servicios ambientales en manglares en México. Cuadernos de Biodiversidad, 54: 41-62.

Góchez, A. (2015). Ciudad con la mayor tasa de crecimiento en Latinoamérica. Recuperado en noviembre 24, 2016, disponible en http://www.razon.com.mx/spip. php?article 277373

Guido Aldana, P., Ramírez Camperos, A., Godínez Orta, L., Cruz León, S. \& Juárez León, A. (2009). Estudio de la erosión costera en Cancún y la Riviera Maya, México. Avances en Recursos Hidraúlicos, 20: 41-56

Hernández Arana, H. (2007). Calentamiento global y zonas costeras: peligrosa combinación. Ecofronteras, 31: 10-13.

Hirales-Cota, M., Espinoza-Avalos, J., Schmook, B., Ruiz-Luna, A. \& Ramos-Reyes, R. (2010). Drivers of mangrove deforestation in Mahahual-Xcalak, Quintana Roo, southeast Mexico. Ciencias Marinas, 36(2): 147159.

INEGI (2015). Encuesta Intercensal 2015. Recuperado en noviembre 27, 2018, disponible en http://www. beta.inegi.org.mx/proyectos/enchogares/especiales/ intercensal 
INEGI (2016a). Censos y conteos de población y vivienda. Recuperado en junio 21, 2017, disponible en http:// www.beta.inegi.org.mx/proyectos/ccpv/2010/default. html

INEGI (2016b). Número de habitantes de Quintana Roo en 2015. Recuperado en junio 21, 2017, disponible en http://cuentame.inegi.org.mx/monografias/ informacion/qroo/poblacion/

IPCC (2014). Fifth Assessment Report of the Intergovernmental Panel on Climate Change. Coastal systems and low-lying areas. Recuperado en noviembre 30, 2018, disponible en https://www.ipcc.ch/pdf/assessmentreport/ar5/wg2/WGIIAR5-Chap5_FINAL.pdf

Knutson, T.R., McBride, J.L., Chan, J., Emanuel, K., Holland, G. Landsea, C., Held, I., Kossin, J.P., Srivastava, A.K., \& Sugi, M. (2010). Tropical Cyclones and Climate Change. Nature Geosci 3.3: 157-163.

Liceaga-Correa, M.A., Arellano Méndez, L.U. \& HernándezNuñez, H. (2010). Efectos de los huracanes y cambio climático sobre el Caribe mexicano: adaptabilidad de los pastos marinos. En: Botello, A.V., VillanuevaFragoso, S., Gutiérrez, J. \& Rojas Galaviz, J.L. (eds.), Vulnerabilidad de las zonas costeras mexicanas ante el cambio climático. SEMARNAT-INE, UNAM-ICMyL, Universidad Autónoma de Campeche. Campeche, México. pp. 211-228

Márquez García, A.Z., Márquez García, E., Bolongaro Crevenna Recasens, A. \& Torres Rodríguez, V. (2010). Cambio en la línea de costa en la Rivera Maya debido a fenómenos hidrometeorológicos extremos, ¿consecuencia del cambio global climático? En: Botello, A.V., Villanueva-Fragoso, S., Gutiérrez, J. \& Rojas Galaviz, J.L. (eds.), Vulnerabilidad de las zonas costeras mexicanas ante el cambio climático. SEMARNAT-INE, UNAM-ICMyL, Universidad Autónoma de Campeche. Campeche, México. pp. 345-358.

Martell-Dubois, R., Mendoza-Baldwin, E., Mariño-Tapia, I., Silva-Casarín, R. \& Escalante-Mancera, E. (2012). Impactos de corto plazo del huracán Dean sobre la morfología de la playa de Cancún, México. Tecnologías y Ciencias del Agua, 3(4): 89-111.

Miranda Jácome, G. (2014). 40 momentos en la historia turística de Cancún. Recuperado en octubre 15, 2016, disponible en http://www.24-horas.mx/40-momentosen-la-historia-turistica-de-cancun-2/

Moguel, R. (2013). Erosión de playas afecta a hoteles de Cancún. Recuperado en abril 13, 2016, disponible en http://sipse.com/novedades/erosion-en-playas-decancun-32656.html
Municipio de Solidaridad (2016a). Ayuntamiento. Medio Físico: orografía, hidrografía, clima, ecosistemas. Recuperado en octubre 18, 2016, disponible en http:// www.municipiodesolidaridad.gob.mx/index.php/ ayuntamiento/medio-fisico

Municipio de Solidaridad (2016b). Ayuntamiento. Historia: Primeros pobladores, Nace el municipio de Solidaridad: 28 de julio de 1993, Solidaridad a sus primeros 20 años. Recuperado en octubre 18, 2016, disponible en http:// www.municipiodesolidaridad.gob.mx/index.php/ ayuntamiento/historia

NOAA (2016). National Hurricane Center. Atlantic. Recuperado en noviembre 28, 2016, disponible en www.nhc.noaa.gov/

Ortiz Pérez, M.A. \& Méndez Linares, A.P. (1999). Escenarios de vulnerabilidad por ascenso del nivel del mar en la costa mexicana del Golfo de México y el Mar Caribe. Investigaciones Geográficas, 39: 68-81.

Pasch, R.J., Blake, E.S., Cobb III, H.D., Roberts, D.P. (2006). Tropical Cyclone Report Hurricane Wilma. 15-25 October 2005. Recuperado en diciembre 14, 2016, disponible en http://www.nhc.noaa.gov/data/tcr/ AL252005_Wilma.pdf

Pérez, T. (2017). Reverdece el mangle a un año de su devastación en Tajamar. Recuperado en enero 16, 2017, disponible en http://sipse.com/novedades/ reverdece-el-mangle-a-un-ano-de-su-devastacionen-tajamar-ecocidio-malecon-dano-ambientalistascancun-238750.html

Ponce-Vélez, G., Villanueva-Fragoso, S. \& García Ruelas, C. (2011). Vulnerabilidad de la zona costera. Ecosistemas costeros del Golfo y Caribe Mexicanos. En: Botello, A.V., Villanueva-Fragoso, S., Gutiérrez, J. \& Rojas Galaviz, J.L. (eds.), Vulnerabilidad de las zonas costeras mexicanas ante el cambio climático. Universidad Autónoma Metropolitana-Iztapalapa, UNAM-ICMyL, Universidad Autónoma de Campeche. Campeche, México. pp. 37-72.

Programa de Desarrollo Urbano de Playa del Carmen (2010). Programa de Desarrollo Urbano del Centro de Población Playa del Carmen, Municipio Solidaridad 20102050. Recuperado en noviembre 28, 2018, disponible en: http://seduvi.qroo.gob.mx/pdus/25PDU\%20 CENTRO \% 20 D E \% 20 P O B L A C I O N \% 20 PLAYA\%20DEL\%20CARMEN\%2020\%20DIC\%20 2010.pdf 
Rosengaus Moshinsky, M., Jiménez Espinosa, M. \& Vázquez Conde, M.T. (2014). Atlas Climatológico de Ciclones Tropicales en México. D.F, México: Centro Nacional de Prevención de Desastres e Instituto Mexicano de Tecnología del Agua. 106 pp.

Ruiz-Ramírez, J.D., Euán-Ávila, J.I. \& Torres-Irineo, E. (2014). Tendencias del nivel del mar en las costas del Caribe mexicano. European Scientific Journal, 10(20): 86-96.

Sánchez, A. (2009). Se agudiza la falta de arena en Cancún. Recuperado en febrero 25, 2016, disponible en http:// www.wradio.com.mx/noticias/actualidad/se-agudiza-lafalta-de-arena-en-cancun/20091124/nota/914775.aspx

Scott, D., Simpson, M.Ch. \& Sim, R. (2012). The vulnerability of Caribbean coastal tourism to scenarios of climate change related sea level rise. Journal of Sustainable Tourism, 20 (6): 883-898

SEDETUR (2017). Reporte anual de turismo Quintana Roo 2017. Secretaría de Turismo. Gobierno del Estado de Quintana Roo 2016-2022. 35 p. Recuperado en noviembre 30, 2018, disponible en: http:// caribemexicano.travel/ARCHIVOS/REPORTE\%20 TURISMO\%202017.pdf.

SEDETUR (2018). Resultados de temporada vacacional, fin de ańo 2017-2018. Secretaría de Turismo. Gobierno del Estado de Quintana Roo 2016-2022. 15 p. Recuperado en noviembre 27, 2018, disponible en: http:// sedeturqroo.gob.mx/ARCHIVOS/RESULTADOSTEMPORADA-FIN-DE-AN\%CC\%83O-2017.pdf.

SEMARNAT (2003). Norma Oficial Mexicana NOM-022SEMARNAT-2003, Que establece las especificaciones para la preservación, conservación, aprovechamiento sustentable y restauración de los humedales costeros en zonas de manglar. Recuperado en marzo 12, 2017, disponible en http://dof.gob.mx/nota_detalle.php?codi go $=697013 \&$ fecha $=10 / 04 / 2003$

SEMARNAT (2010). Norma Oficial Mexicana NOM059-SEMARNAT-2010, Protección ambiental-Especies nativas de México de flora y fauna silvestres-Categorías de riesgo y especificaciones para su inclusión, exclusión o cambio-Lista de especies en riesgo. Recuperado en marzo 12, 2017, disponible en http://dof.gob.mx/nota detalle_popup.php?codigo $=5173091$

Shepard, C.C., Agostini, V.N., Gilmer, B., Allen, T., Stone, J., Brooks, W. \& Beck, M. (2012). Assessing future risk: quantifying the effects of sea level rise on storm surge risk for the southern shores of Long Island, New York. Natural Hazards, 60(2): 727-745
Torres Alvarado, M.R. \& Calva Benítez, L. (2011). Costa Maya Sur y cambio climático: posible efecto del huracán Dean en la calidad ambiental. En: Botello, A.V., Villanueva-Fragoso, S., Gutiérrez, J. \& Rojas Galaviz, J.L. (eds.), Vulnerabilidad de las zonas costeras mexicanas ante el cambio climático. Universidad Autónoma Metropolitana-Iztapalapa, UNAM-ICMyL, Universidad Autónoma de Campeche. Campeche, México. pp. 675-698.

Universidad de Colorado (2017). Global mean sea level rates. Recuperado en junio 8, 2017, disponible en http://sealevel.colorado.edu/content/regional-sea-leveltime-series

Valderrama-Landeros, L.H., Rodríguez-Zúñiga, M.T., Troche-Souza, C., Velázquez-Salazar, S., VilledaChávez, E., Alcántara-Maya, J.A., Vázquez-Balderas B., Cruz-López, M.I. \& Ressl, R. (2017). Manglares de México: actualización y exploración de los datos del sistema de monitoreo 1970/1980-2015. Comisión Nacional para el Conocimiento y Uso de la Biodiversidad. Ciudad de México, 128 pp.

Vázquez Botello, A. (2008). Evaluación regional de la vulnerabilidad actual y futura de la zona costera mexicana y los deltas más impactados ante el incremento del nivel del mar debido al cambio climático y fenómenos hidrometeorológicos extremos. D.F. México: Instituto Nacional de Ecología, Universidad Nacional Autónoma de México y Secretaría de Medio Ambiente y Recursos Naturales. 121 pp.

Verdayes Ortiz, F. (2013). Historia general de Cancún (de la Prehistoria al 43 aniversario). Recuperado en febrero 20, 2016, disponible en http://www.revistapioneros. $\mathrm{com} /$ historia-general-de-cancun-de-la-prehistoria-al43-aniversario/

Wong, P.P., Losada, I.J., Gattuso, J.P., Hinkel, J., Khattabi, A., McInnes, K.L., Saito, Y. \& Sallenger, A. (2014). Coastal systems and low-lying areas. In: Climate Change 2014: Impacts, Adaptation, and Vulnerability. Part A: Global and Sectoral Aspects. Contribution of Working Group II to the Fifth Assessment Report of the Intergovernmental Panel on Climate Change [Field, C.B., V.R. Barros, D.J. Dokken, K.J. Mach, M.D. Mastrandrea, T.E. Bilir, M. Chatterjee, K.L. Ebi, Y.O. Estrada, R.C. Genova, B. Girma, E.S. Kissel, A.N. Levy, S. MacCracken, P.R. Mastrandrea, and L.L. White (eds.)]. Cambridge University Press, Cambridge, United Kingdom and New York, NY, USA. pp 361-409.

Yañez-Arancibia, A., Twilley, R.R. \& Lara Domínguez, A.L. (1998). Los ecosistemas de manglar frente al cambio climático global. Madera y bosques, 4(2): 3-19. 
Este número ha recibido una ayuda del Vicerrectorado de Investigación y Transferencia de Conocimiento de la Universidad de Alicante
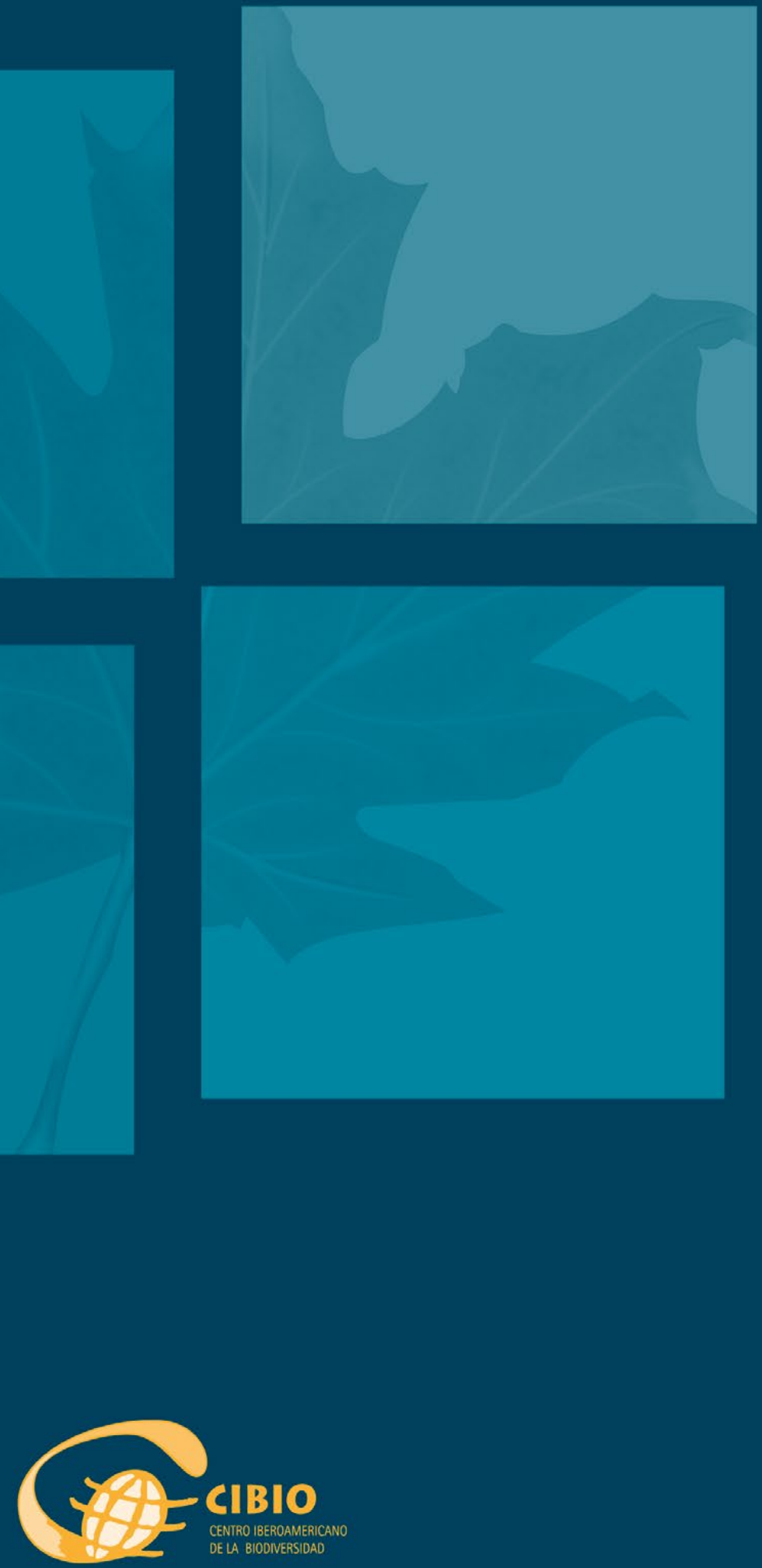

\section{Universitat d'Alacant
Universidad de Alicante}

